\title{
APLICAÇÃO DA LÓGICA FUZZY PARA ESTUDO DE PERMEABILIDADE DE SOLOS DE REGIÃO IMPACTADA DA BAIXADA SANTISTA
}

\section{APPLICATION OF FUZZY LOGIC FOR SOIL PERMEABILITY STUDY OF THE IMPACTED REGION OF THE SANTOS REGION}

\section{Sandra Regina Monteiro Masalskiene Roveda ${ }^{1}$, José Arnaldo Frutuoso Roveda ${ }^{2}$, Roberto Wagner Lourenço ${ }^{3}$}

\author{
${ }^{1}$ Matemática, Profa. Dra. Engenharia Ambiental, Universidade Estadual Paulista, \\ Campus Sorocaba. UNESP. e-mail: sandra@sorocaba.unesp.br \\ ${ }^{2}$ Matemático, Prof. Dr. Engenharia Ambiental, Universidade Estadual Paulista, \\ Campus Sorocaba. UNESP. e-mail: roveda@sorocaba.unesp.br \\ ${ }^{3}$ Geógrafo. Prof. Dr. Engenharia Ambiental, Universidade Estadual Paulista, Campus \\ Sorocaba. UNESP. e-mail: robertow@sorocaba.unesp.br
}

\begin{abstract}
RESUMO
A poluição do solo tem sido cada vez mais um motivo de preocupação para toda a sociedade e para as autoridades. É um tema complexo que envolve questões políticas, econômicas e também dificuldades técnicas. Um aspecto de relevante interesse são os mecanismos de permeabilidade cujo conhecimento é bastante importante na análise de impactos ambientais em diversos contextos. $\mathrm{O}$ propósito deste trabalho foi estudar a permeabilidade de solos de região impactada da Baixada Santista utilizando sistemas de inferência fuzzy. A metodologia proposta foi aplicada em dados oriundos de coletas localizadas nas áreas urbanas e periféricas dos centros administrativos de parte da Baixada Santista. Como resultado são mostrados dois mapas de permeabilidade da região estudada, obtidos por métodos de defuzzificação distintos, são eles o centróide e a média dos máximos. Observa-se que tais mapas, apesar de apresentarem características de permeabilidade distintas, estão de acordo com o que se espera de uma região litorânea, mostrando assim que a metodologia adotada é viável para este tipo de estudo.
\end{abstract}

Palavras-chave: Lógica fuzzy. Permeabilidade. Sistemas fuzzy.

\begin{abstract}
Soil pollution has increasingly been a concern for society and the authorities. It is a complex issue that involves political, economic and also technical difficulties. One aspect of relevant interest are the mechanisms of permeability whose knowledge is very important in the analysis of environmental impacts in different contexts. The purpose of this work was to study the permeability of soils impacted region of Santos using fuzzy inference systems. The proposed methodology was applied to data from collections located in urban and administrative centers of the peripheral part of Santos. As a result two maps of permeability of the studied region are shown, obtained by different
\end{abstract}


methods of defuzzification, centroid and the average maximum. It is observed that such maps, although with different permeability characteristics are in agreement with what is expected of a coastal region, thus showing that the methodology is viable for this type of study.

Keywords: Fuzzy logic. Permeability. Fuzzy sistems.

\section{INTRODUÇÃO}

A poluição do solo tem sido cada vez mais um motivo de preocupação para toda a sociedade e para as autoridades. É um tema complexo que envolve questões políticas, econômicas e também dificuldades técnicas. Sob a perspectiva de que o solo é um componente fundamental no ecossistema mundial, o conceito de protegê-lo tem sido objeto de intensas discussões e estudos no cenário mundial (BRAGA, 2002).

Um aspecto de relevante interesse são os mecanismos de permeabilidade cujo conhecimento é bastante importante na análise de impactos ambientais em diversos contextos, tais como modelagem de fluxo subterrâneo, determinação das propriedades hidráulicas de água de lixiviação em áreas de disposição de resíduos, cálculo da compressibilidade do solo entre outros.

Embora equações empíricas sejam usadas para estimar o parâmetro de permeabilidade (FREEZE e CHERRY, 1979; III CARRIER, 2003; CHAPUIS, 2004), essas equações têm certas limitações e incertezas (SEZER, 2009). Por esse motivo Sezer sugere uma metodologia alternativa de cálculo da permeabilidade utilizando à lógica fuzzy.

Esta teoria matemática, introduzida por Zadeh (1965), permite expressar incertezas e subjetividades já que podem ser usadas informações qualitativas e quantitativas. A vagueza decorrente dos termos linguísticos (muito, pouco, baixo, alto, etc.) tradicionalmente vistas como não-científicas são admissíveis na abordagem fuzzy aproximando muitas vezes a representação do sistema à própria realidade.

O propósito deste trabalho foi estudar a permeabilidade de solos de região impactada da Baixada Santista utilizando sistemas de inferência fuzzy. A metodologia proposta foi aplicada em dados oriundos de coletas localizadas nas áreas urbanas e periféricas dos centros administrativos de parte da Baixada Santista.

\section{MATERIAL E MÉTODOS}

\subsection{Modelagem Fuzzy}

Nesta seção será descrito o processo de modelagem utilizado para calcular a permeabilidade do solo em função de suas propriedades físicas. As variáveis de entrada consideradas são: declividade e quantidade de argila, silte, areia e matéria orgânica. Para a modelagem foi usado o processo de inferência fuzzy, que utiliza três conceitos importantes: funções de pertinência, operações entre conjuntos fuzzy e base de regras (BARROS e BASSANEZI, 2006), que são definidos a seguir.

Definição 1: A função de pertinência de um conjunto fuzzy $F$ é denotada por:

$$
\mu_{F}: X \rightarrow[0,1]
$$

onde $X$ denota um conjunto universo que é sempre um conjunto clássico e $F$, um conjunto fuzzy, subconjunto de $X$. Para cada $x \in X$, o valor $\mu_{F}(x)$ indica o grau de pertinência do elemento $x$ no conjunto fuzzy $F$, sendo que para $\mu_{F}(x)=0, x$ não pertence ao conjunto e $\mu_{F}(x)=1$ indica que $x$ pertence completamente. 
Assim, um subconjunto fuzzy $F$ é composto de elementos $x$ de um conjunto clássico $X$, providos de um valor de pertinência a $F$, dado por $\mu_{F}(x)$. Pode-se dizer que um subconjunto fuzzy $F$ de $X$ é dado por um conjunto (clássico) de pares ordenados:

$$
F=\left\{\left(x, \mu_{F}(x)\right), x \in X\right\} .
$$

A escolha da função de pertinência depende do modelador e/ou do contexto analisado. Entre as formas de uma função de pertinência tem-se a triangular e a trapezoidal como as mais utilizadas. Neste estudo optou-se pela função trapezoidal, que é dada por:

$$
\mu_{F}(x)=\left\{\begin{array}{cc}
\frac{x-a}{b-a} & \text { se } a \leq x<b \\
1 \quad & \text { se } b \leq x \leq c \\
\frac{d-x}{d-c} & \text { se } c<x<d \\
0 \quad \text { caso contrário }
\end{array}\right.
$$

Foram construídos cinco conjuntos de funções de pertinência que expressam as variáveis de entrada, como conjuntos fuzzy mostrados nas Figuras 1 e 2, observe que para areia e argila tem-se o mesmo conjunto de funções de pertinência.
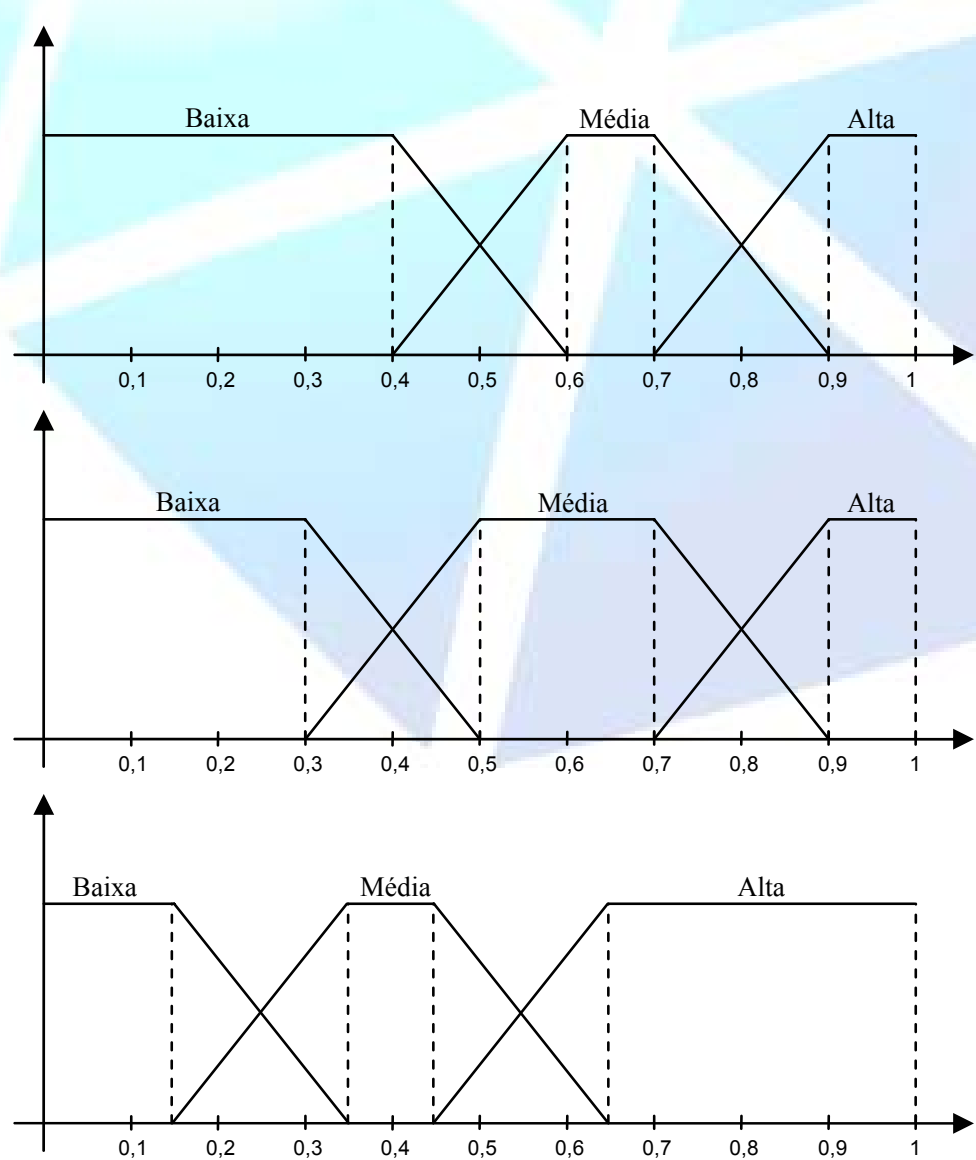

Figura 1. Funções de pertinência para silte, matéria orgânica e declividade 


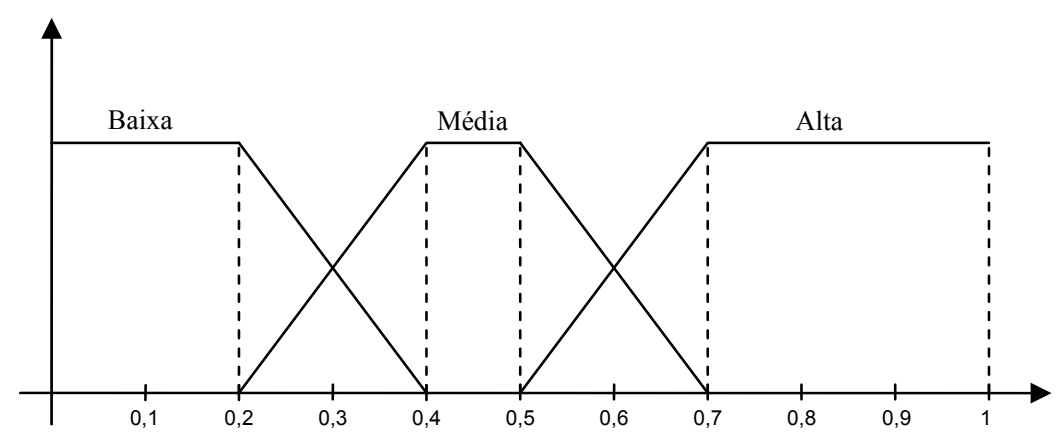

Figura 2. Funções de pertinência para areia e argila

A Figura 3 mostra um conjunto de funções para a variável de saída, a permeabilidade.

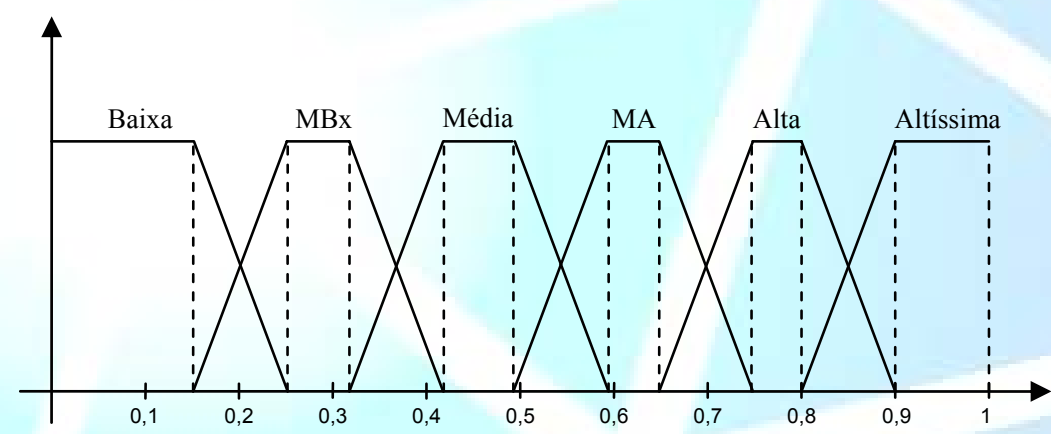

Figura 3. Funções de pertinência para permeabilidade

As operações padrões de conjuntos fuzzy são: intersecção (operador and), união (operador or) e complementar de subconjuntos fuzzy (operador not). Essas são operações para subconjuntos fuzzy agregados, ou seja, dados dois ou mais conjuntos fuzzy, estes produzem um único subconjunto fuzzy, isto é, um agregado dos subconjuntos dados. Essas operações são essenciais na natureza da lógica fuzzy.

Definição 2: Sejam $F$ e $G$ subconjuntos fuzzy de $X$. A intersecção entre $F$ e $G$ é o subconjunto de $X$ dado pela função de pertinência:

$$
\mu_{(F \cap G)}(x)=\min _{x \in X}\left\{\mu_{F}(x), \mu_{G}(x)\right\} .
$$

A união entre $F$ e $G$ é o subconjunto fuzzy de $X$ cuja função de pertinência se denota por:

$$
\mu_{(F \cup G)}(x)=\max _{x \in X}\left\{\mu_{F}(x), \mu_{G}(x)\right\} .
$$

O complementar do subconjunto $F$ é o subconjunto fuzzy $F$ ' de $X$ que é dado por:

$$
\mu_{F^{\prime}}(x)=1-\mu_{F}(x), \quad x \in X .
$$


No processo de inferência fuzzy, as variáveis linguísticas desempenham um papel muito importante, pois determinam as relações entre as variáveis de entrada e de saída, sendo estas matematicamente estabelecidas pelas regras associadas aos processos. Uma regra "se-então" tem a forma "Se $x$ é $A$ então $y$ é $B$ ", onde $A$ e $B$ são valores linguísticos modelados pelos conjuntos $f u z z y$. O acompanhante do termo "se" é chamado de antecedente e o do termo "então", consequente. O conjunto dessas regras é denominado base de regras.

Com a ajuda de um especialista, que levou em consideração cada uma das variáveis de entrada e suas medidas de interferência na permeabilidade do solo, foram construídas 66 regras para este estudo. Elencamos abaixo três das proposições utilizadas neste sistema:

Regra 2: Se a declividade é baixa, quantidade de areia é baixa, quantidade de Matéria Orgânica é baixa, quantidade de argila é alta e quantidade de silte é alta, então a permeabilidade do solo é baixa.

(...)

Regra 53: Se a declividade é baixa, quantidade de areia é alta, quantidade de Matéria Orgânica é alta, quantidade de argila é baixa e quantidade de silte é baixa, então a permeabilidade do solo é altíssima.

(...)

Regra 63: Se a declividade é média, quantidade de areia é alta, a quantidade de Matéria Orgânica é alta, quantidade de argila é baixa e quantidade de silte é baixa então a permeabilidade do solo é baixa.

(...)

O método de inferência fuzzy é o mecanismo que avalia matematicamente as informações subjetivas definidas na base de regras. Para essa inferência, há dois métodos bastante utilizados: o de Mamdani e o de Takagi-Sugeno. As regras "se-então", explicadas anteriormente, são definidas pelo produto cartesiano fuzzy dos conjuntos fuzzy que compõem o antecedente e o consequente da regra.

Aqui, foi utilizado o Método de Mamdani que agrega as regras utilizando o operador or, ou seja, operador máximo, e em cada uma das regras é utilizado o operador and, operador mínimo (Figura 4).

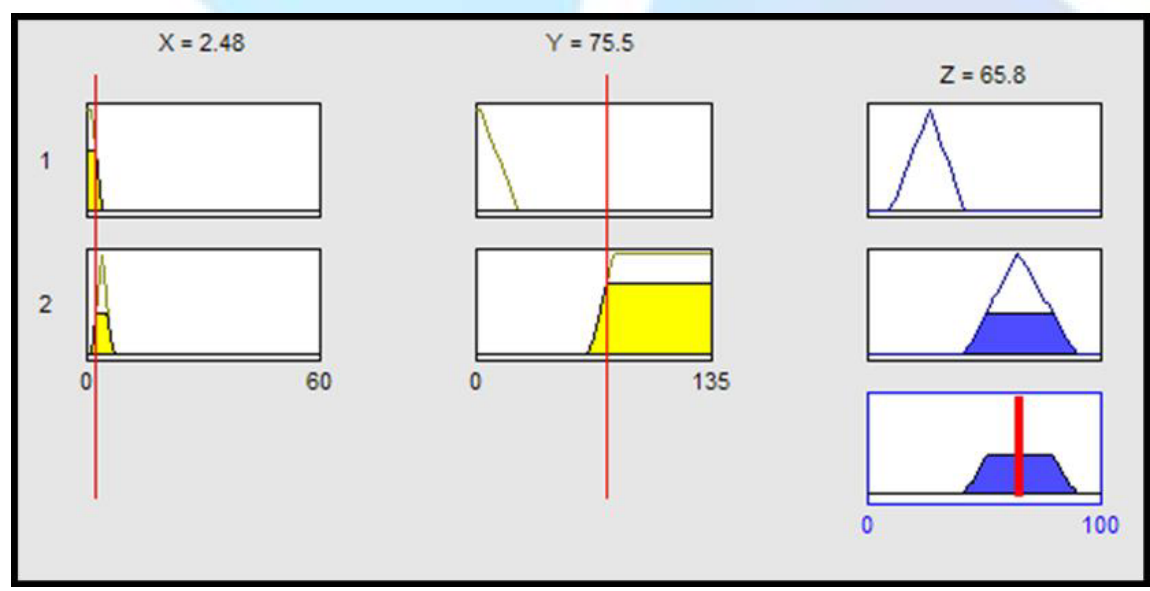

Figura 4. Ilustração do Método Mamdani 
Finalmente, a defuzzificação, última parte do processo, consiste em transformar a saída fuzzy em um valor numérico crisp, ou seja, em um número real. Entre os métodos de defuzzificação mais conhecidos, destacam-se: centro de gravidade, centro dos máximos, média dos máximos.

Nesse estudo foi utilizado a média dos máximos e o centro de gravidade.

\section{2 Área de Estudo}

A Folha de Santos de projeção U.T.M, cobre uma área de aproximadamente $794 \mathrm{~km}^{2}$, entre

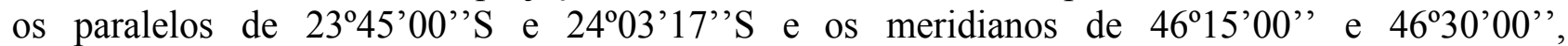
compreendendo extensa área da região denominada Baixada Santista, na Faixa Atlântica do Estado de São Paulo. Os principais municípios da Região da Baixada Santista são: Santos e Cubatão (inteiramente representados), Guarujá, Praia Grande e São Vicente, com suas respectivas sedes e, também, parte predominantemente rural, de alguns municípios da Região da Grande São Paulo Ribeirão Pires, Rio Grande da Serra, Santo André e São Bernardo do Campo (Figura 5).

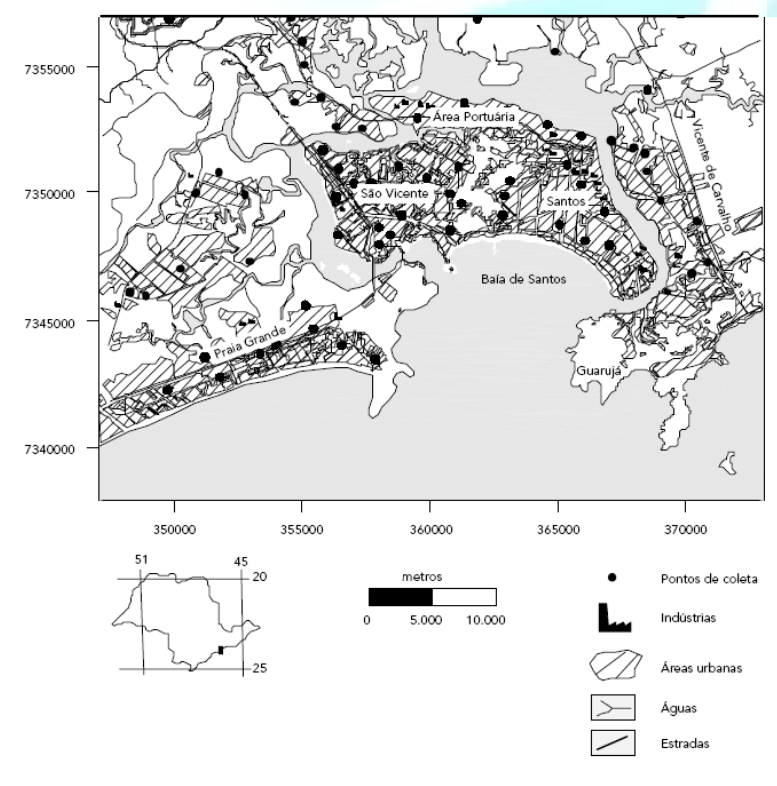

Figura 5. Municípios da Baixada Santista

\section{RESULTADOS E DISCUSSÃO}

Os dados de entrada são provinentes de amostras obtidas a partir da coleta de solo em áreas com e sem influência antropogênica (LOURENÇO, 2002). Foram considerados 455 pontos com informações sobre a quantidade de argila, silte e areia, além da matéria orgânica e declividade do solo em questão. Para defuzzificação dos conjuntos fuzzy de permeabilidade obtidos foi utilizado o método do centróide (ou centro de gravidade) e o método da média dos máximos, a fim de comparar os resultados obtidos. As Figuras 6 e 7 mostram a variação de permeabilidade na área de estudo. 


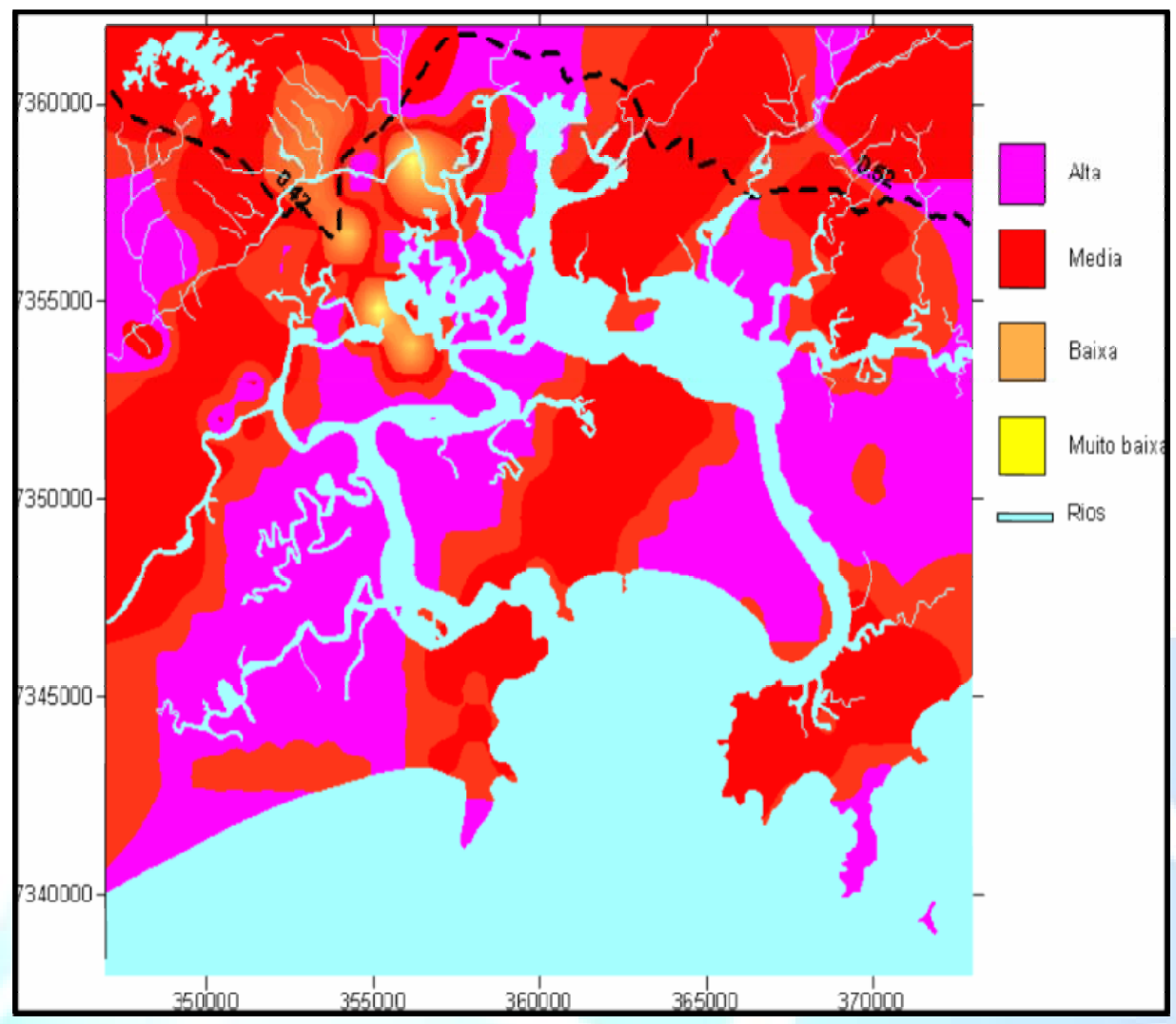

Figura 6. Mapa de permeabilidade - Processo de Defuzzificação: centróide

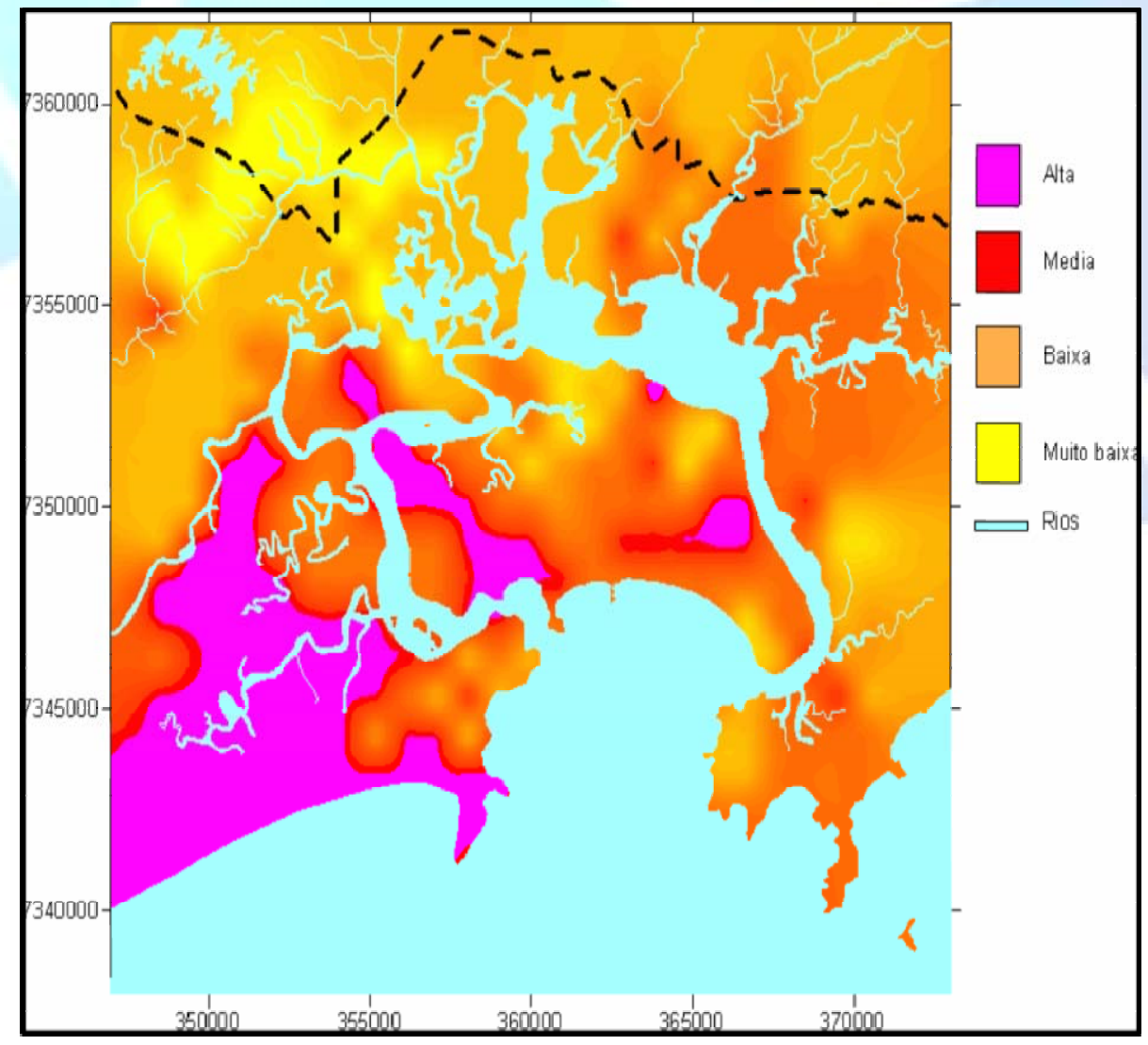

Figura 7. Mapa de permeabilidade - Processo de Defuzificação: Média dos Máximos 
A análise dos mapas gerados apresenta uma maior variabilidade da permeabilidade na Figura 7. Observe que isso se deve ao fato da escolha dos métodos de defuzificação utilizados. Na Figura 6 o método escolhido foi o centróide que se assemelha à média ponderada para distribuição de dados, porém os pesos são valores $\varphi\left(x_{i}\right)$, indicando o grau de compatibilidade do $x_{i}$ com a modelagem do conjunto fuzzy. Portanto, esse método faz a média de todas as figuras que representam os graus de pertinência de um subconjunto fuzzy, fazendo com que os resultados obtidos sejam mais suaves do que aqueles encontrados pelo método da média dos máximos.

\section{CONCLUSÕES}

Pode-se observar que ambos os mapas, apesar de apresentarem características de permeabilidade diferentes, estão de acordo com o que se idealiza de uma área litorânea, que é o fato de esperarmos regiões mais permeáveis próximo do litoral, em virtude de mangues e terreno arenoso e de regiões menos permeáveis quando termina a planície costeira e começa a região mais montanhosa, com pedras e menos areia, coerente com as características físicas, geológicas e pedológicas de solo e de rocha.

Dessa forma, a metodologia descrita mostra-se viável para este tipo de estudo de permeabilidade.

\section{REFERÊNCIAS}

BARROS, L.C.; BASSANEZI, R.C. Tópicos de Lógica Fuzzy e Biomatemática. Campinas: Comissão de Publicações IMECC, 2006.

BRAGA, B.; HESPANHOL I.; CONEJO, J.G.L; BARROS, M.T.L.; SPENCER, M.; PORTO, M.; NUCCI, N.; JULIANO, N.; EIGER, S. Introdução à Engenharia Ambiental. São Paulo: Prentice Hall, 2002.

CARRIER III W.D. Goodbye, Hazen, Hello, kozeny-Carman. Journal of Geotechnical and Geoenvironmental Engineering - ASCE, vol.129, p.1054-1056, 2003.

CHAPUIS, R P. Predicting the saturated Hydraulic conductivity of sand and gravel using effective diameter and void ratio. Canadá Geotechnical Journal, vol. 41, p.787-795, 2004.

FREEZE R. A. E.; CHERRY J A. Groundwater. Prentice Hall, New Jersey, 1979.

LOURENÇO, R.W. Modelagem Espacial e Modelos Ambientais Dinâmicos em SIG. 2002. 213f. Tese (Doutorado em Geociências) - Universidade Estadual Paulista - UNESP, Rio Claro SP, 2002.

SEZER, A; GOKTEPE, A B; ALTUN, A. Estimation of the permeability of granular soils using neuro-fuzzy system, AIAI Workshops Proceedings, 2009. 